\title{
Mycobacterium tuberculosis septum site determining protein, Ssd encoded by rv3660c, promotes filamentation and elicits an alternative metabolic and dormancy stress response
}

Kathleen England ${ }^{1,2}$, Rebecca Crew ${ }^{1}$ and Richard A Slayden ${ }^{1 *}$

\begin{abstract}
Background: Proteins that are involved in regulation of cell division and cell cycle progression remain undefined in Mycobacterium tuberculosis. In addition, there is a growing appreciation that regulation of cell replication at the point of division is important in establishing a non-replicating persistent state. Accordingly, the objective of this study was to use a systematic approach consisting of consensus-modeling bioinformatics, ultrastructural analysis, and transcriptional mapping to identify septum regulatory proteins that participate in adaptive metabolic responses in M. tuberculosis.

Results: Septum site determining protein (Ssd), encoded by rv3660c was discovered to be an ortholog of septum site regulating proteins in actinobacteria by bioinformatics analysis. Increased expression of ssd in M. smegmatis and M. tuberculosis inhibited septum formation resulting in elongated cells devoid of septa. Transcriptional mapping in M. tuberculosis showed that increased ssd expression elicited a unique response including the dormancy regulon and alternative sigma factors that are thought to play a role in adaptive metabolism. Disruption of $r v 3660 \mathrm{c}$ by transposon insertion negated the unique transcriptional response and led to a reduced bacterial length.

Conclusions: This study establishes the first connection between a septum regulatory protein and induction of alternative metabolism consisting of alternative sigma factors and the dormancy regulon that is associated with establishing a non-replicating persistent intracellular lifestyle. The identification of a regulatory component involved in cell cycle regulation linked to the dormancy response, whether directly or indirectly, provides a foundation for additional studies and furthers our understanding of the complex mechanisms involved in establishing a nonreplicating state and resumption of growth.
\end{abstract}

Keywords: Mycobacterium tuberculosis dormancy, Dos regulon, septum site determining protein, cell division

\section{Background}

Despite effective chemotherapeutic regimens, Mycobacterium tuberculosis remains one of the most significant public health problems, with an estimated global burden of one third of the world's population. The unremitting global burden is attributed, in part, to the ability of $M$. tuberculosis to establish and maintain a non-replicating

\footnotetext{
* Correspondence: richard.slayden@colostate.edu

1 Mycobacteria Research Laboratories, Department of Microbiology, Immunology, and Pathology. Colorado State University, Fort Collins, CO 80523, USA

Full list of author information is available at the end of the article
}

persistent infection, thus making the bacillus tolerant to drug treatment and host immune response [1,2]. Studies have demonstrated that the development of non-replicating persistence involves a shift from rapid to slow growth followed by a complete shutdown of cell cycle progression characterized by a complete round of DNA replication and inhibition of cell division [3-5]. These experimental observations indicate that cell division, and septum formation in particular, is a key regulatory checkpoint of the cell cycle for entry into a non-replicating state. However, proteins that regulate septum
C Biomed Central

() 2011 England et al; licensee BioMed Central Ltd. This is an Open Access article distributed under the terms of the Creative Commons Attribution License (http://creativecommons.org/licenses/by/2.0), which permits unrestricted use, distribution, and reproduction in any medium, provided the original work is properly cited. 
formation as part of growth arrest and altered metabolic responses associated with the persistent state remain undefined in M. tuberculosis. Thus, it is important to identify regulatory elements involved in septum formation and the cell cycle in context of adaptive metabolism and to the development of a non-replicating persistent state.

Cell cycle progression in bacteria, including $M$. tuberculosis, is governed in response to stress conditions substantiating the notion that septum regulation and cell division events are regulated under a variety of circumstances [6-10]. Response and adaption to stress is a complex series of events that relies on coordination of multiple processes. The prototypical stress response is the SOS response, which involves check-point regulation and de-repression of genes under direct and indirect control of a common repressor. Eliciting the SOS response leads to a cessation in cell division due to inhibition of FtsZ polymerization via SulA, and transient induction of alternative functions [11,12]. In addition to DNA repair, there are other mechanisms that are controlled by the SOS response, thus establishing that responses to stress share common components with regards to regulation. Similarly, in $M$. tuberculosis inhibition of FtsZ polymerization and cell division occurs in response to stress conditions, which include environmental changes that occur during pathogenesis and drug treatment. Therefore, inhibition of septum formation through the regulation of Fts $\mathrm{Z}$ polymerization represents a common mechanism that is conserved among bacteria, including $M$. tuberculosis, to control cell division and cell cycle activity in response to various conditions including stress [8].

In model organisms, FtsZ polymerization is controlled under normal growth conditions by a variety of FtsZ interacting regulatory elements including Minsystem proteins, Div proteins, MipZ and under stress conditions by proteins such as SulA [13]. In Gramnegative organisms septum site selection and regulation are controlled by the Min-system consisting of MinC, MinD and MinE, while in Gram-positive organisms the system consists of MinC, MinD, and an ortholog DivIVa. Along with these proteins, other proteins that have a demonstrated regulation in FtsZ polymerization have been identified; however the precise role these regulatory components play is not well defined. One group of FtsZ regulatory proteins is the septum site determining proteins. This family of proteins has limited similarity to proteins involved in morphological differentiation in Streptomyces spp. These components work together to negatively regulate FtsZ polymerization preventing cell division until DNA replication is complete and the chromosomes have been properly segregated.
It is well accepted that during establishment of a chronic latent infection $M$. tuberculosis halts cell cycle progression and significantly reduces metabolic activity. One adaptive process that has been associated with limited growth conditions, stress, and pathogenesis is the Dos-response. Under experimental conditions, the Dos regulon is induced in response hypoxia, $\mathrm{NO}$ and carbon monoxide [14]. The Dos-response is generally thought to be important for adaptation to alternative growth conditions, thus establishing the ability to endure long periods within the host. The idea that the Dos-response plays a role in pathogenesis is supported by studies that have demonstrated that the highly virulent W-Beijing linage of $M$. tuberculosis exhibits high levels of constitutive expression of the Dos-regulon components $[15,16]$. While the DosR two-component regulatory system and primary members of the Dos-regulon are well defined, other components, particularly complimentary regulatory elements that coordinate cell cycle progression and growth in response to alternative growth conditions remain undefined. Because bioinformatics approaches alone have failed to identify homologs for all cell cycle components, we have previously used inhibition of cell division and transcriptional mapping to identify putative regulatory elements in $M$. tuberculosis, with particular focus on those that regulate septum formation $[6,7,17]$.

The detailed regulatory mechanisms involved in inhibition of septum formation and cell division in $M$. tuberculosis have not been defined, and will afford an understanding of the mechanisms involved with growth and adaptation to alternative environments signaling the induction of bacteria into a non-replicating state. In order to identify septum regulatory proteins that elicit a transcriptional stress response, a systematic approach consisting of consensus-modeling bioinformatics, gene dosage and ultrastructural analysis, and expression profiling was employed. As a result, rv3660c was discovered to encode a protein with similarity to the loosely defined family of septum site determining proteins. Increased expression of $r v 3360 c$ resulted in filamentous cells, while the disruption of the gene by transposon insertion presented minicell morphology demonstrating an inhibitory role in septum formation. Transcriptional analysis showed that rv3660c expression results in the induction of a unique profile of alternative sigma factors, open reading frames encoding proteins involved in alternative metabolism and the dormancy regulon. Accordingly, this is the first report of a Ssd-like septum regulating protein in $M$. tuberculosis, and that stalls cell division and is associated with induction of alternative metabolism associated with pathogenesis and survival of nonreplicating bacilli, thus representing a previously unidentified regulatory mechanism in M. tuberculosis. These data, in combination with previous studies to identify 
septum regulatory elements in $M$. tuberculosis, indicate that the protein encoded by rv3360c is Ssd, a septum site determining protein.

\section{Results \\ rv3660c encodes a previously unidentified septum site determining-like protein, Ssd}

A bioinformatics approach utilizing consensus sequences derived from global alignments of annotated MinD proteins (OMA Group 78690) and septum site determining proteins (OMA Group 73337) was taken to search the $M$. tuberculosis H37Rv genome for open reading frames that encode putative MinD-like and Ssdlike orthologs. The search using the Ssd consensus identified the conserved hypothetical open reading frame $r v 3660 c$, which is consistent with previous bioinformatics and experimental assignment. Search of the $M$. tuberculosis genome with the MinD consensus sequence also identified $r v 3660 c$, but with less similarity to MinD orthologs with $30 \%$ sequence similarity. Identification of Rv3660c using both Ssd and MinD consensus models strongly indicates that $r v 3660 c$ encodes a FtsZ regulatory protein. Alignments of the protein encoded by $r v 3660 c$ with the MinD and Ssd consensus sequences confirmed and substantiated that the protein encoded by $r v 3660 c$ is a member of the septum site determining protein family (Figure 1). Further evidence that rv3660c encoded a Ssd protein was obtained from hierarchical clustering analysis of Ssd encoded by $r v 3660 c, 46$ proteins annotated as MinD and 37 proteins annotated as
Ssd. Hierarchical clustering analysis resulted in SsD (Rv3660c) grouping with Ssd proteins encoded in actinobacteria. This data is consistent with previous data that, $r v 3660 c$ was mapped to septum formation in transcriptional mapping studies [6].

\section{ssd expression promotes filamentation in $M$. smegmatis and M. tuberculosis}

To assess if Ssd inhibits septum formation in mycobacteria, gene dosage studies were conducted in M. smegmatis and M. tuberculosis, and bacterial ultrastructure was visualized and measured by scanning electron microscopy (Figure 2). The expression of $s s d$ in merodiploid strains was assessed by quantitative RT-PCR and production was confirmed by western blot analysis. Expression of ssd was more robust in M. smegmatis than $M$. tuberculosis as compared to SigA expression. In the $M$. tuberculosis merodiploid strain ssd expression was 10-20 fold increased on average over endogenous expression levels. These studies also revealed that $s s d$ is constitutively expressed at low levels throughout the growth cycle under laboratory growth conditions. This observation is consistent with the oberserved low level expression of other stress responses $[14,16]$. There was no significant difference in the growth rate or physical characteristics, such as clumping or pigmentation between $M$. smegmatis and $M$. tuberculosis strains expressing ssd and control strains. The primary distinguishing physical feature between the M. smegmatis and M. tuberculosis ssd expressing merodiploid strains in

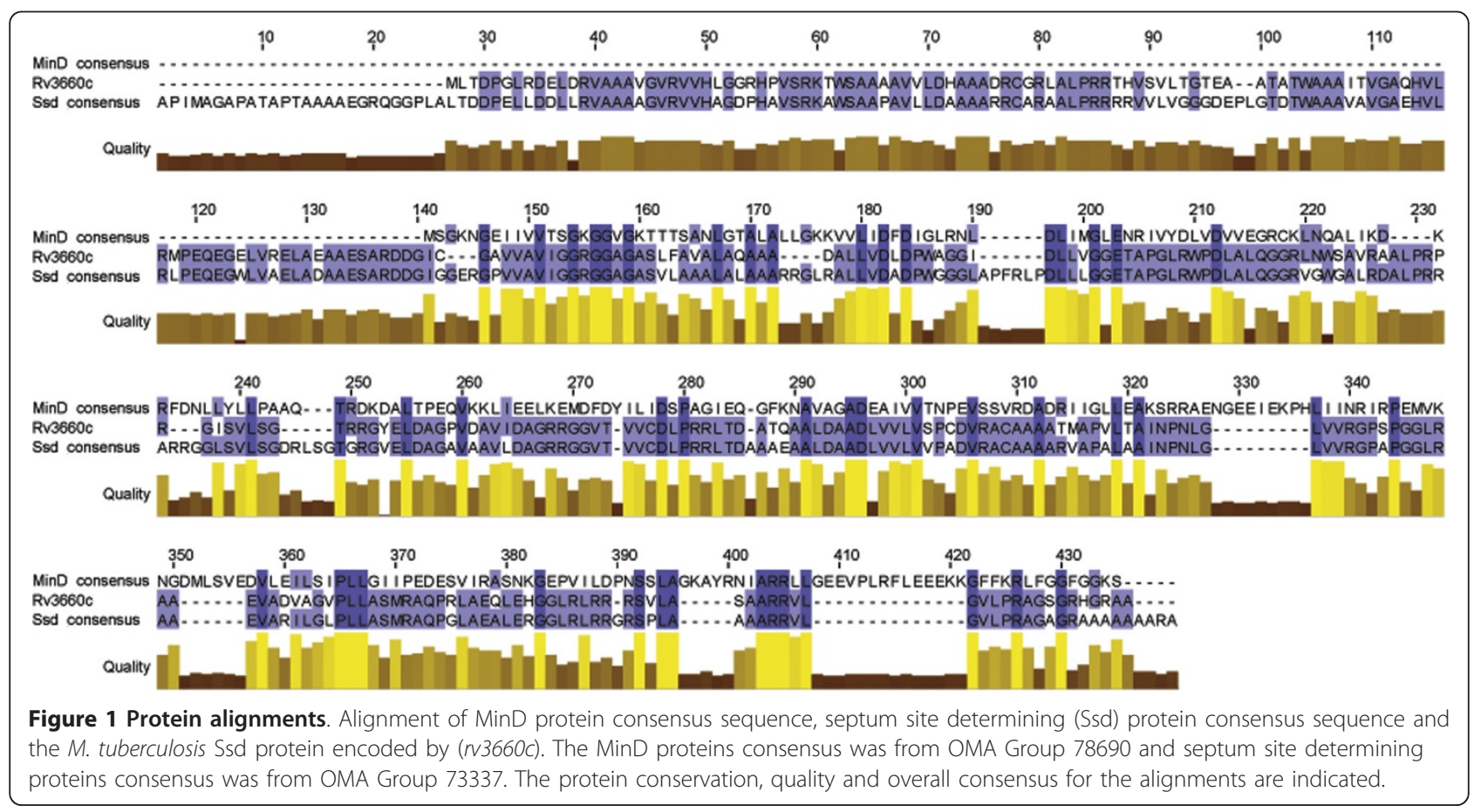



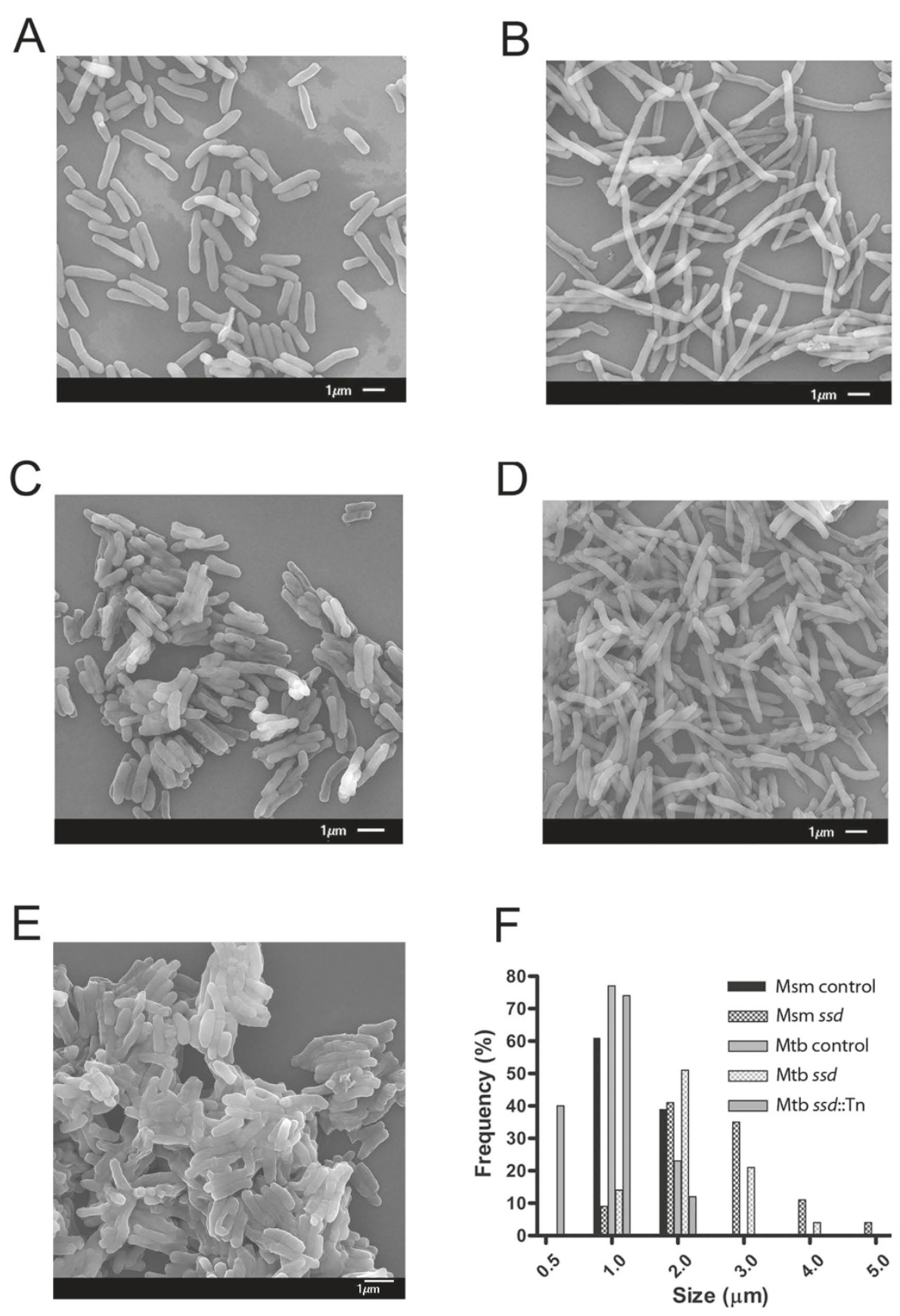

Figure 2 Ultrastructure Analysis (SEM) and Length distributions. Bacterial morphology. (A) M. smegmatis control strain, (B) M. smegmatis ssd merodiploid (C) M. tuberculosis control, (D) M. tuberculosis ssd merodiploid and (E) ssd::Tn mutant M. tuberculosis strain were visualized by scanning electron microscopy. Images are representative of different fields of bacteria from exponentially growing cultures at $37^{\circ} \mathrm{C}$. (F) Lengths of the bacterial cells were calculated from the coordinates of both ends of the cell as measured from representative fields as visualized by scanning electron microscopy. Multiple fields were examined and values calculated in 0.5-1 mm increments from multiple fields of over 100 cells.

comparison to control bacteria was increased cell lengths and a smooth ultrastructural characteristic (Figure 2ABCD). The observed smooth ultrastructure devoid of concentric rings along the bacterial filament is important because this observation is consistent with inhibition of FtsZ polymerization and Z-ring formation as previously reported $[6,7,17,18]$. The $M$. smegmatis wild type control strain exhibited cell lengths of $2.1 \pm$ $0.11 \mu \mathrm{m}$ (Figure 2AF) and the M. smegmatis ssd merodiploid strain had increased cell lengths of $3.2 \pm 0.42 \mu \mathrm{m}$ 
(Figure 2BF). Similarly, M. tuberculosis H37Rv control cells had lengths of $1.73 \pm 0.43 \mu \mathrm{m}$ (Figure $2 \mathrm{CF}$ ) and expression of $s s d$ resulted in increased cell lengths of $2.53 \pm 0.76 \mu \mathrm{m}$ (Figure 2DF). In contrast, a ssd::Tn $M$. tuberculosis mutant strain had decreased cell lengths of $1.35 \pm 0.51 \mu \mathrm{m}$ (Figure 2EF). This experimental data demonstrates a causal relationship between the expression levels of $s s d$ and altered bacterial cell lengths, confirming the bioinformatics analysis and further substantiating Ssd as a septum regulation protein as annotated (http://genolist.pasteur.fr/TubercuList[19]) and indicated by transcriptional mapping [6]

\section{Whole-genome expression profiling of $s s d$ merodiploid and mutant strains}

To assess the effect of ssd expression on $M$. tuberculosis metabolism, global gene expression profiling was performed on the ssd overexpression $M$. tuberculosis merodiploid strain. A total of 2,274 ORFs were transcriptionally active with 432 of these ORFs being differentially expressed 1.5 -fold or greater change ( $\mathrm{p}$ values $\leq 0.05$ ). Overall, genes with altered transcription encode proteins involved in lipid metabolism, cell respiration, protein synthesis, cell wall surface molecules, cell cycle progression, and most notably genes involved in dormancy and stress.

The genes induced to the greatest extent as a result of increased $s s d$ expression were alternative sigma factors and members of the dosR-regulon and (Table 1). The dosR-dependent genes (rv3131, hspX and tgs1) and the alternative sigma factors (sigF, sigG, sigH sigI, sigJ, sigL and $\operatorname{sig} M$ ) along with genes involved in adaptive metabolic functions such as anaerobic respiration ( $\mathrm{frdAB}$, nirBD, narl, narJ, narG, narU, narX and narK2), electron transport and redox-potential (ackA, fprB, cydC, $c y d B, \operatorname{app} C, f d x A$, and $r u b A)$, and genes associated with fatty acid degradation ( $f a d$, ech, acc, $m u t$ ) were induced. In additional to the increased expression of genes involved in adaptive metabolism and stress, the $s s d$ merodiploid induced the expression of polyketide genes $p k s 6-11,17$ and 18 and various lipoprotein genes $l p p$ and lpq (Table 2). These genes are also associated with adaptive responses to alternative growth conditions and have been shown to contribute to virulence traits in $M$. tuberculosis [20]. In contrast, genes encoding ribosomal proteins ( $r p l, r p s, r p m)$ required for protein synthesis were downregulated. These transcriptional activities are concordant with increased transcriptional activity of genes involved in dormancy, adaptive responses, and conditions associated with a non-replicating persistent lifestyle.

To determine whether the observed dos-response was a direct result of $s s d$ expression, transcriptional analysis of the ssd::Tn mutant $M$. tuberculosis strain was performed. Compared to the ssd merodiploid strain, only 65 genes displayed a 1.5 -fold or greater ( $\mathrm{p}$ values $\leq$ 0.05 ) change in expression in the $s s d$ mutant. Of notable absence in the transcriptional response in the $s s d:: \operatorname{Tn}$ mutant strain are genes of the dos-regulon the other stress associated genes, and the virulence-associated genes that were identified in the ssd overexpressing mutant strain. The observed limited number of differentially expressed genes includes those involved in the cell cycle processes of lipid biosynthesis (kasA and kasB), the chromosome partitioning gene parA, and the divIVa homologue, wag31. Notably, parA, and the divIVa are known to be involved in regulation and coordination of chromosome partitioning and septum placement events, which is consistent with a mild disruption in coordination of chromosome partitioning and cell division. Thus, the contrasting and unique induction of the dos-regulon, alternative sigma factors and virulence genes upon $s s d$ overexpression indicates that these responses result from increased levels of $s s d$ and are connected to regulatory events involved in septum formation.

The differentially expressed dos-regulated genes, cell cycle discriminant genes and sigma factors identified by microarray were validated by quantitative RT-PCR analysis (Figure 3). The concordance in expression trends of these genes as determined by microarray and quantitative RT-PCR specifically verify that $s s d$ expression induced genes of the dos-regulon and stress genes (Figure 3A), with altered expression of cell cycle genes (Figure $3 \mathrm{~B}$ ), all of which are consistent with septum inhibition. With regards to the sigma factors, sigA expression was repressed in the $s s d$ merdodiploid strain while the alternative sigma factors $\operatorname{sigF}$, sigG, sigH. sigI, sigJ, sigL and sigM were induced (Figure 3C). The quantitative RT-PCR analysis was concordant with the expression trends observed by microarray and confirmed that $s s d$ expression elicits a $d o s R$-like stress response consisting of known dos-members and alternative sigma factors, which was not observed in the $s s d$ mutant.

\section{Discussion}

M. tuberculosis is able to circumvent host responses and establish a latent infection where it can silently persist for years. While the bacterial response to growth in various environments has been reported, the proteins that participate in the complex regulatory processes that govern growth in response to stress or changing environments remain largely unknown. Proteins that are orthologs of know septum formation regulatory elements are candidates for participating in non-replicating persistence because the reversible "off" and "on" regulation allows relapse of disease. Accordingly, a consensus sequence modeling approach was employed to identify putative septum formation inhibitors and, genes dosage 
Table 1 dos $R$ regulon gene expression from transcriptional profiles of $s s d$ merodiploid strain and the ssd::Tn mutant strain

\begin{tabular}{|c|c|c|c|c|c|c|c|}
\hline Locus & Gene & Product & merodiploid & & mutant & & $\Delta$ \\
\hline & & & $\log _{2} \exp$ & $p$-value & $\log _{2} \exp$ & $p$-value & \\
\hline Rv0079 & & hypothetical protein & 1.31 & 0.007 & 0.27 & 0.000 & 4.9 \\
\hline Rv0080 & & hypothetical protein & 1.35 & 0.002 & 0.20 & 0.001 & 6.7 \\
\hline Rv0081 & & transcriptional regulator (ArsR family) & 1.10 & 0.000 & 0.20 & 0.016 & 5.4 \\
\hline Rv0082 & & probable oxidoreductase subunit & 0.46 & 0.011 & 0.28 & 0.063 & 1.7 \\
\hline Rv0083 & & probable oxidoreductase subunit & 0.10 & 0.001 & 0.88 & 0.008 & 0.1 \\
\hline Rv0569 & & conserved hypothetical protein & 1.26 & 0.000 & 0.29 & 0.003 & 4.3 \\
\hline Rv0570 & $\operatorname{nrdZ}$ & ribonucleotide reductase, class II & 1.19 & 0.018 & -0.08 & 0.003 & -15.0 \\
\hline Rv0571c & & conserved hypothetical protein & 0.14 & 0.025 & -0.15 & 0.000 & -0.9 \\
\hline Rv0572C & & hypothetical protein & 0.30 & 0.002 & -0.41 & 0.013 & -0.7 \\
\hline Rv0573c & & conserved hypothetical protein & 0.83 & 0.006 & 0.19 & 0.000 & 4.4 \\
\hline Rv0574c & & conserved hypothetical protein & 0.76 & 0.009 & -0.23 & 0.006 & -3.2 \\
\hline Rv1733c & & possible membrane protein & 1.99 & 0.068 & 0.33 & 0.002 & 6.0 \\
\hline Rv1734c & & hypothetical protein & 0.71 & 0.013 & -0.04 & 0.009 & -18.0 \\
\hline Rv1735c & & hypothetical protein & 0.50 & 0.001 & 0.14 & 0.012 & 3.4 \\
\hline Rv1736c & narX & fused nitrate reductase & 1.09 & 0.032 & 0.07 & 0.000 & 15.0 \\
\hline Rv1737c & nark2 & nitrite extrusion protein & 1.87 & 0.228 & 0.20 & 0.001 & 9.2 \\
\hline Rv1738 & & conserved hypothetical protein & 2.90 & 0.230 & 0.96 & 0.016 & 3.0 \\
\hline Rv1812C & & probable dehydrogenase & 0.03 & 0.324 & -0.15 & 0.001 & -0.2 \\
\hline Rv1813c & & conserved hypothetical protein & 1.26 & 0.257 & 1.83 & 0.030 & 0.7 \\
\hline Rv1996 & & conserved hypothetical protein & 2.63 & 0.046 & 0.80 & 0.025 & 3.3 \\
\hline Rv1997 & $c t p F$ & probable cation transport ATPase & 1.62 & 0.001 & 0.17 & 0.018 & 9.4 \\
\hline Rv1998c & & conserved hypothetical protein & 0.47 & 0.118 & 0.10 & 0.000 & 4.6 \\
\hline Rv2003c & & conserved hypothetical protein & 1.26 & 0.004 & 0.08 & 0.010 & 15.1 \\
\hline Rv2004c & & hypothetical protein & 1.01 & 0.008 & 0.36 & 0.022 & 2.8 \\
\hline Rv2005c & & conserved hypothetical protein & 1.78 & 0.033 & 0.33 & 0.000 & 5.4 \\
\hline Rv2006 & otsB2 & trehalose-6-phosphate phosphatase & 1.28 & 0.000 & 0.02 & 0.008 & 78.4 \\
\hline Rv2007c & $f d x A$ & ferredoxin & 2.56 & 0.137 & 0.64 & 0.026 & 4.0 \\
\hline Rv2027c & dost & sensor histidine kinase & 1.35 & 0.001 & 0.07 & 0.044 & 18.9 \\
\hline Rv2028c & & conserved hypothetical protein & 0.38 & 0.009 & -0.11 & 0.004 & -3.3 \\
\hline Rv2029c & $p f k B$ & phosphofructokinase II & 2.03 & 0.330 & 0.26 & 0.006 & 7.8 \\
\hline Rv2030c & & conserved hypothetical protein & 3.37 & 0.195 & 0.62 & 0.004 & 5.4 \\
\hline Rv2031c & hspX & $14 \mathrm{kD}$ antigen, heat shock protein Hsp20 family & 3.94 & 0.043 & 1.50 & 0.079 & 2.6 \\
\hline Rv2032 & acg & conserved hypothetical protein & 2.50 & 0.277 & 0.29 & 0.003 & 8.6 \\
\hline Rv2617c & & hypothetical protein & -0.21 & 0.012 & -0.01 & 0.000 & 20.6 \\
\hline Rv2623 & & conserved hypothetical protein & 3.02 & 0.151 & 0.15 & 0.132 & 19.8 \\
\hline Rv2624c & & conserved hypothetical protein & 1.34 & 0.062 & 0.10 & 0.024 & 13.9 \\
\hline Rv2625c & & conserved hypothetical protein & -0.03 & 0.016 & -0.94 & 0.017 & 0.0 \\
\hline Rv2626c & & conserved hypothetical protein & 3.35 & 0.000 & 0.77 & 0.184 & 4.4 \\
\hline Rv2627c & & conserved hypothetical protein & 2.65 & 0.285 & 0.05 & 0.010 & 51.0 \\
\hline Rv2628 & & hypothetical protein & 2.22 & 0.022 & 0.14 & 0.038 & 16.0 \\
\hline Rv2629 & & hypothetical protein & 0.49 & 0.004 & 0.28 & 0.006 & 1.8 \\
\hline Rv2630 & & hypothetical protein & 1.42 & 0.003 & 0.24 & 0.014 & 5.9 \\
\hline Rv2631 & & conserved hypothetical protein & 0.70 & 0.015 & -0.17 & 0.021 & -4.1 \\
\hline Rv2830c & & similar to phage P1 phd gene & 0.29 & 0.000 & -0.07 & 0.002 & -3.9 \\
\hline Rv3126c & & hypothetical protein & 0.91 & 0.021 & 0.07 & 0.018 & 12.8 \\
\hline
\end{tabular}


Table 1 dos $R$ regulon gene expression from transcriptional profiles of ssd merodiploid strain and the ssd::Tn mutant strain (Continued)

\begin{tabular}{|c|c|c|c|c|c|c|c|}
\hline Rv3127 & & conserved hypothetical protein & 2.15 & 0.044 & 0.51 & 0.000 & 4.2 \\
\hline Rv3128c & & conserved hypothetical protein & 0.30 & 0.310 & 0.13 & 0.002 & 2.3 \\
\hline Rv3129 & & conserved hypothetical protein & 1.09 & 0.002 & 0.03 & 0.035 & 40.6 \\
\hline Rv3130c & $\operatorname{tgs} 1$ & conserved hypothetical protein & 3.92 & 0.309 & 0.84 & 0.013 & 4.7 \\
\hline Rv3131 & & conserved hypothetical protein & 4.01 & 0.273 & 1.66 & 0.189 & 2.4 \\
\hline Rv3132c & doss & sensor histidine kinase & 2.00 & 0.014 & 0.18 & 0.001 & 11.0 \\
\hline Rv3133c & dosR & two-component response regulator & 1.00 & 0.070 & 0.22 & 0.009 & 4.5 \\
\hline Rv3134c & & conserved hypothetical protein & 2.45 & 0.024 & 0.16 & 0.002 & 15.0 \\
\hline Rv3841 & $b f r B$ & bacterioferritin & 1.22 & 0.106 & 1.36 & 0.087 & 0.9 \\
\hline
\end{tabular}

studies were performed to assess the morphological characteristics and global transcriptional profiling to assess the effect on the transcriptional response of cell cycle and metabolism components.

Alignments with Ssd and MinD consensus sequences, and clustering analysis with Ssd and MinD proteins demonstrated that the protein encoded by $r v 3660 c$ has similarity to $S s d$-family proteins. Visualization of the $M$. smegmatis and $M$. tuberculosis ssd merodiploid strains and $M$. tuberculosis ssd::Tn mutant strain by scanning electron microscopy demonstrated a link between the abundance of Ssd and an elongated morphology. Bacterial filamentation is known to occur in M. tuberculosis and other bacteria when cell division is inhibited $[7,17,18,21]$. In addition, in $M$. tuberculosis visualization of the ultrastructure of the bacterial filaments reveals information about whether the inhibition is early or late in the cell division process $[6,7,17,18]$. When septum formation in $M$. tuberculosis is inhibited the resulting bacterial filaments are smooth and largely devoid of concentric rings indicative of established septal sites that arise when cell division is inhibited at later steps. This is an important ultrastructural distinction because inhibition of cell division at the stage of septum formation has been associated with entry into non-replicating persistence and associated with growth in macrophages [22]. Therefore, the observation that the ssd merodiploid strains of either M. smegmatis or M. tuberculosis displays a filamentous morphology devoid of septa is consistent with inhibition of septum formation, a characteristic associated with in vivo growth [22]. In addition to $r v 3660 c$ being annotated as encoding a septum site determining protein it has also been associated experimentally with altered septum formation via inhibition of FtsZ polymerization and transcriptional mapping [6]. These results are fully consistent with being a putative septum site-determining protein.

Coincident with the altered growth and morphology, the M. tuberculosis ssd merodploid strain exhibited an adaptive genetic program that has been associated with survival and virulence. Reports of transcriptional profiles of $M$. tuberculosis exposed to a variety of conditions thought to model the in vivo growth environment including hypoxia, nutrient starvation, and murine infection revealed a set of common genes of the $\operatorname{dos} R$ regulon and those involved in lipid metabolism, cell wall maintenance and remodeling, and alternative respiration and redox balance [14,23-28]. When gene expression in the $M$. tuberculosis ssd merodiploid strain was evaluated, it was found that in conjunction with induction of the $\operatorname{dos} R$ regulon there was a $D o s$-like response characterized by an upregulation of genes involved in fatty acid degradation, anaerobic respiration, electron transport or redox-potential, and a down-regulation of ribosomal proteins and protein synthesis. Importantly, in the $s s d$ mutant, these genes did not display a significant difference in transcriptional activity, indicating that Ssd plays a role in Dos-regulation and cellular adaptation under unique environmental conditions along with septum regulation.

In addition to the Dos-response, increased expression of $s s d$ resulted in an induction of a unique alternative sigma factor response. The responsive sigma factors have been associated with adaptation to environmental stresses and virulence $[29,30]$. SigF has been associated with phosphate uptake, antibiotic treatment and drug tolerance [31-33]. SigG and SigH are known to be induced under stress conditions associated with DNA damage and heat and oxidative-stress responses, respectively [33,34]. SigI is directly upregulated by SigJ expression, which controls an alternative $\mathrm{H}_{2} \mathrm{O}_{2}$ resistance pathway for survival in the macrophage [35]. Other sigma factors such as SigL and SigM are thought to be involved in remodeling of the bacterial cell surface and production of proteins such as esat6-homologs that are necessary for survival and persistence in animal models of tuberculosis that closely mimic human infection $[36,37]$. Since it has been proposed that the role of these rarely expressed alternative sigma factors are related to host-specific conditions then the unique profile elicited 
Table 2 Genes differentially regulated for selected cell functions ( $p$-value $\leq \mathbf{0 . 0 5}$ )

\begin{tabular}{|c|c|c|c|c|c|c|c|}
\hline ORF & Gene & $\log _{2}$ expression & & ORF & Gene & $\log _{2}$ expression & \\
\hline & & merodiploid & mutant & & & merodiploid & mutant \\
\hline \multicolumn{4}{|c|}{ Fatty acid utilization } & \multicolumn{4}{|c|}{ Ribosomal proteins } \\
\hline Rv0974c & accD2 & 1.2 & -0.2 & Rv0056 & rpll & -1.0 & -0.6 \\
\hline Rv1935c & echA13 & 0.9 & -0.2 & Rv0682 & rpsL & -0.9 & -0.9 \\
\hline Rv2486 & echA14 & 1.0 & -0.1 & Rv0700 & rps」 & -1.4 & -0.5 \\
\hline Rv0456c & echA2 & 1.2 & -0.1 & Rv0701 & $r p / C$ & -1.5 & -0.4 \\
\hline Rv3550 & echA20 & 1.1 & 0.2 & Rv0716 & rplE & -1.2 & -0.9 \\
\hline Rv0971c & echA7 & 1.3 & -0.1 & Rv0722 & $r p m D$ & -0.9 & -0.3 \\
\hline Rv3546 & fadA5 & 1.1 & 0.1 & Rv0723 & rp/O & -0.7 & -0.2 \\
\hline Rv1715 & fadB3 & 1.0 & -0.1 & Rv2441c & rpmA & -0.9 & -0.5 \\
\hline Rv0099 & fadD10 & 1.2 & 0.0 & Rv3442C & rpsl & -0.9 & -0.2 \\
\hline Rv1550 & fadD11 & 1.0 & 0.2 & Rv3443c & $r p / M$ & -1.6 & -0.5 \\
\hline Rv1058 & fadD14 & 1.2 & 0.0 & Rv3458c & $r p s D$ & -0.8 & -0.5 \\
\hline Rv3561 & fadD3 & 0.8 & 0.5 & Rv3460c & $r p s M$ & -1.3 & -0.6 \\
\hline Rv0035 & fadD34 & 1.3 & 0.0 & Rv3461c & rpm」 & -1.4 & -0.6 \\
\hline Rv0214 & fadD4 & 0.8 & -0.2 & Rv3924c & rpmH & -1.2 & -0.7 \\
\hline Rv0551c & fadD8 & 0.9 & 0.0 & & & & \\
\hline Rv2590 & fadD9 & 1.3 & -0.5 & \multicolumn{4}{|c|}{ Anaerobic respiration } \\
\hline Rv0972C & fadE12 & 1.4 & -0.1 & Rv0252 & nirB & 0.8 & $\mathrm{ndr}$ \\
\hline Rv0975c & fadE13 & 1.3 & $\mathrm{ndr}$ & Rv0253 & nirD & 1.1 & $n d r$ \\
\hline Rv3061c & fadE22 & 1.0 & -0.1 & Rv0267 & narU & 1.2 & ndr \\
\hline Rv3505 & fadE27 & 1.0 & 0.0 & Rv1161 & narG & 0.7 & $\mathrm{ndr}$ \\
\hline Rv3544c & fadE28 & 0.8 & 0.0 & Rv1163 & narJ & 0.7 & $\mathrm{ndr}$ \\
\hline Rv3562 & fadE31 & 1.0 & 0.2 & Rv1164 & narl & 0.6 & ndr \\
\hline Rv3563 & fadE32 & 0.8 & 0.4 & Rv1552 & frdA & 1.2 & $\mathrm{ndr}$ \\
\hline Rv3564 & fadE33 & 1.2 & 0.3 & Rv1553 & $\operatorname{frdB}$ & 0.8 & $\mathrm{ndr}$ \\
\hline Rv0752C & fadE9 & 0.9 & -0.1 & Rv1554 & frdC & 1.1 & $\mathrm{ndr}$ \\
\hline Rv1492 & mutA & 1.1 & 0.2 & Rv1736c & narX & 1.1 & $\mathrm{ndr}$ \\
\hline Rv1493 & mutB & 1.2 & 0.5 & Rv1737c & nark2 & 1.9 & 0.2 \\
\hline
\end{tabular}

\begin{tabular}{|c|c|c|c|c|c|c|c|}
\hline \multicolumn{4}{|c|}{ Cell surface molecules } & \multicolumn{4}{|c|}{ Electron Trpt/Redox } \\
\hline Rv0399c & IpqK & 0.8 & -0.1 & Rv0409 & ackA & 1.0 & 0.2 \\
\hline Rv0405 & pks6 & 1.2 & -0.2 & Rv0886 & fprB & 0.8 & 0.1 \\
\hline Rv0593 & IprL & 1.1 & 0.0 & Rv1620c & cydC & 1.6 & 0.0 \\
\hline Rv0604 & IpqO & 0.8 & -0.1 & Rv1622C & $c y d B$ & 2.0 & -0.2 \\
\hline Rv0794c & $I p d B$ & 0.9 & -0.2 & Rv1623c & $a p p C$ & 1.0 & -0.2 \\
\hline Rv1064c & IpqV & 1.1 & -0.1 & Rv2007c & $f d x A$ & 2.6 & 0.6 \\
\hline Rv1166 & IpqW & 0.8 & 0.0 & Rv3251c & rubA & 0.8 & -0.1 \\
\hline Rv1372 & pks18 & 1.1 & 0.1 & & & & \\
\hline Rv1661 & pks7 & 1.3 & -0.2 & ATP synt & & & \\
\hline Rv1662 & pks8 & 1.0 & 0.2 & Rv1304 & $a t p B$ & 0.2 & -0.6 \\
\hline Rv1663 & pks17 & 1.2 & 0.2 & Rv1305 & atpE & 0.2 & -0.4 \\
\hline Rv1664 & pks9 & 1.1 & 0.1 & Rv1306 & atpF & 0.0 & -0.7 \\
\hline Rv1665 & pks11 & 0.7 & 0.2 & Rv1307 & atpH & 0.2 & -0.6 \\
\hline Rv1921c & IppF & 1.4 & 0.2 & Rv1308 & $\operatorname{atp} A$ & 0.3 & -0.4 \\
\hline Rv1946c & IppG & 1.0 & 0.1 & Rv1309 & $\operatorname{atpG}$ & -0.1 & -0.7 \\
\hline Rv1966 & mce3 & 1.1 & 0.0 & Rv1310 & $a t p D$ & 0.3 & -0.4 \\
\hline
\end{tabular}


Table 2 Genes differentially regulated for selected cell functions (p-value ? $\leq$ ? 0.05) (Continued)

\begin{tabular}{lllllll}
\hline Rv2270 & $\operatorname{IppN}$ & 0.9 & -0.1 & Rv1311 & atpC & 0.2 \\
\hline Rv2330c & $\operatorname{IppP}$ & 0.7 & 0.1 & & -0.4 \\
\hline Rv2543 & $\operatorname{IppA}$ & 0.9 & 0.2 & ndr = not differentially regulated & \\
\hline Rv2796c & $\operatorname{IppV}$ & 0.8 & 0.0 & & \\
\hline
\end{tabular}

\section{A}

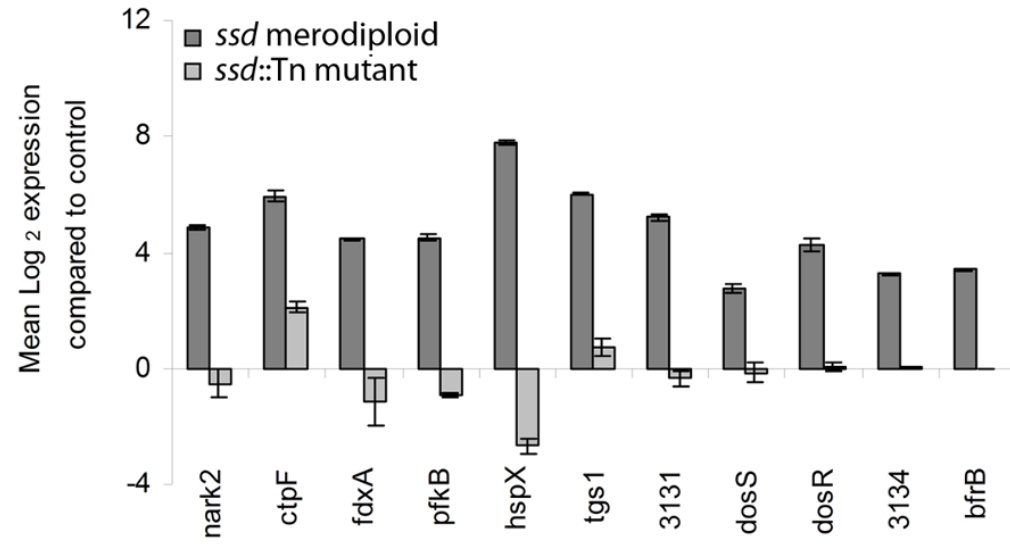

B

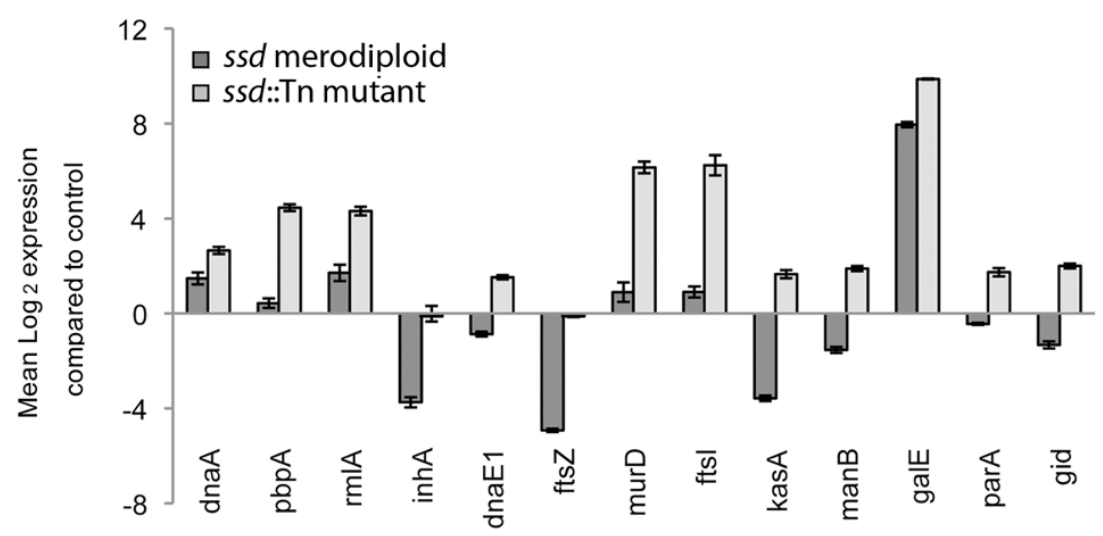

C

4

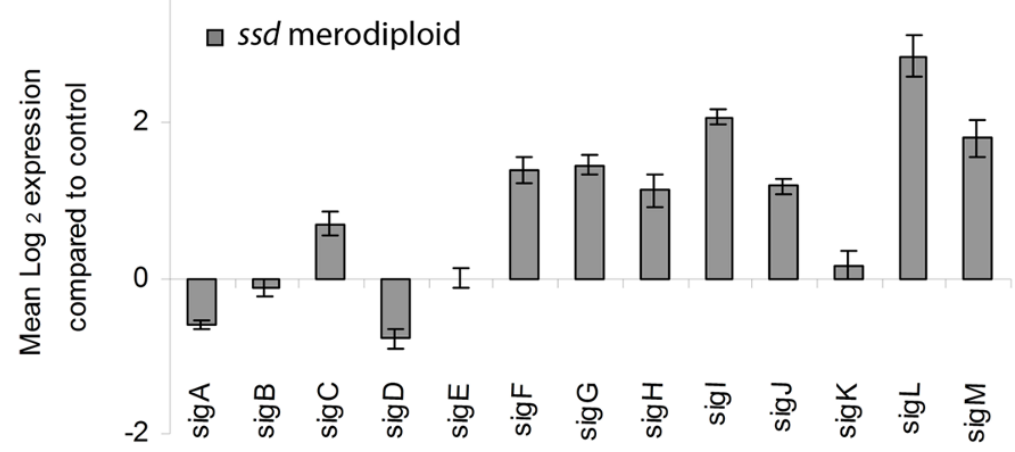

Figure 3 Quantitative real time-PCR analysis of select genes. Mean $\log _{2}$ expression for (A) representative dosR regulon genes, (B) cell cycle discriminant genes and (C) sigma factors in the ssd merodiploid $M$. tuberculosis strain compared to $M$. tuberculosis control strain. Data are mean values \pm SD from independent biological samples. Ratios were calculated using the total number of gene targets from the $s s d$ merodiploid $M$. tuberculosis strain or ssd::Tn mutant M. tuberculosis strain compared to paired M. tuberculosis control stain. 
by increased ssd expression demonstrates a role for Ssd in modulation of septum formation and cell division as part of the global adaptive strategy for survival in the host.

\section{Conclusion}

In order to survive, $M$. tuberculosis must adapt to a stressful intracellular environment, which requires a global alternative adaptive response. Among the adaptive responses, the Dos-response is the best characterized, and has been associated with virulence. In addition to the Dos-regulon, other adaptive responses including regulation of cell division and cell cycle progression are involved in establishing a non-replicating persistent lifestyle. While all the components involved in regulation and metabolic adaptation regarding cessation of growth and non-replicating persistence in $M$. tuberculosis have yet to be defined, the results presented here substantiate Ssd as a component of a global regulatory mechanism that promotes a shift into an altered metabolic state. This is the first report providing evidence linking a regulatory element of septum formation with an adaptive response associated with virulence and non-replicating persistence in $M$. tuberculosis. Clearly, further experimentation is required to elucidate the precise mechanism of action of Ssd in regulating septum formation and its role in adaptive metabolism during stress.

\section{Methods}

\section{Bioinformatic analysis}

To identify putative MinD or septum site determining proteins encoded in M. tuberculosis, a MinD and a Ssd consensus-model sequences was created from alignments of protein sequences annotated as MinD (OMA Group 78690) or as septum site determining proteins (OMA Group 73337) from a variety of bacterial species. The resulting MinD and Ssd consensus model sequences were then used to search and identify proteins encoded in the $M$. tuberculosis genome. In all BLAST searches, the percent identity and score were optimized.

\section{Molecular biology and bacterial strains}

The ssd ( $r v 3660 c$ ) open reading frame was PCR amplified from M. tuberculosis H37Rv genomic DNA using AccuPrime pfx DNA polymerase (Invitrogen) with primer sequences 5'-ctgaccgatccgggg and 3'-gtgccatcccgccgt engineered with asymmetric NdeI and HindIII restriction sites respectively, to facilitate cloning into the extrachromosomal mycobacterial vector $p$ VV16. Transformation into M. tuberculosis $\mathrm{H} 37 \mathrm{Rv}$ and selection were performed as previously described [17]. For all experiments $M$. tuberculosis merodiploid and the rv3660c mutant strain (Tn mutant E150, provided by TBVTRM contract:
HHSN266200400091c) were cultivated at $37^{\circ} \mathrm{C}$ in Middlebrook $7 \mathrm{H} 9$ liquid medium supplemented with $0.2 \%$ glycerol, 10\% OADC (oleic acid, albumin, dextrose and catalase enrichment), and $0.05 \%$ Tween 80 or on supplemented Middlebrook 7H11 agar medium containing 50 $\mu \mathrm{g} / \mathrm{ml}$ kanamycin when necessary.

\section{Ultrastructure analysis by scanning electron microscopy}

For visualization of bacterial ultrastructure by SEM, bacterial cells were washed three times in PBS, $\mathrm{pH} 7.4$, and fixed with $2.5 \%$ gluteraldehyde in Buffer A $(0.1 \mathrm{M}$ potassium phosphate ( $\mathrm{pH} 7.4), 1 \mathrm{mM} \mathrm{CaCl} 2$ and $1 \mathrm{mM}$ $\mathrm{MgCl}_{2}$ ) at $4^{\circ} \mathrm{C}$ for $24 \mathrm{hrs}$. The fixed cells were collected by centrifugation, washed three times in Buffer $\mathrm{A}$ and treated with $1 \% \mathrm{OsO}_{4}$ in Buffer A for 30 minutes at $4^{\circ} \mathrm{C}$. After treatment, cells were washed three times with Buffer A. and prepared for SEM with a graded series of ethanol treatments (20-100\%). Ultrastructure examination was performed using a JOEL JEM -100CX electron microscope.

\section{Global transcriptional profiling}

For transcriptional analysis, three independent biological replicates of $M$. tuberculosis $\mathrm{H} 37 \mathrm{Rv}$ control strain, three independent biological replicates of a $M$. tuberculosis H37Rv ssd merodiploid strain and three independent biological replicates of a $M$. tuberculosis H37Rv ssd::Tn mutant strain were grown to mid-log phase growth $(\mathrm{O}$. D. $600 \mathrm{~nm}=0.3-0.4)$, harvested by centrifugation, and subjected to TRIzol before RNA isolation. Following physical disruption with $0.1 \mathrm{~mm}$ zirconium grinding beads, total RNA was purified using an RNeasy kit (Qiagen) as previously described [6]. Labeled cDNAs were generated using direct labeling from $5 \mu \mathrm{g}$ of total RNA and hybridized to M. tuberculosis whole genome DNA microarrays obtained from the TB Vaccine Testing and Research Materials Contract (HHSN266200400091c) at Colorado State University as described [6]. Slides were scanned with a Genepix 4000B scanner. Global normalization was performed on the raw fluorescent intensities, and each feature of the array (Cy3 and $\mathrm{Cy} 5)$ was normalized to the mean channel intensity and subjected to Anova single factor analysis. Transcriptionally active open reading frames were considered to be those with SNR $>2$ and a $P$ value of $\leq 0.05$. GEO accession \# Pending submission/data release. Self-organizing map (SOM) analysis was performed using all transcriptionally active open reading frames.

\section{Quantitative real-time PCR}

Quantitative real-time PCR was performed on selected open reading frames to verify transcriptional expression found by microarray as described [6]. Quantitative RTPCR primers were designed according using Primer-3 
and analyses were performed using SYBR-green chemistry (Invitrogen). RNA isolation and cDNA preparation was carried out as described above. PCR amplification was performed with a thermocycling program of $55^{\circ} \mathrm{C}$ for $5 \mathrm{~min}$ then $95^{\circ} \mathrm{C}$ for 2 minutes, 45 cycles of $95^{\circ} \mathrm{C}$ for $15 \mathrm{sec}, 60^{\circ} \mathrm{C}$ for $30 \mathrm{sec}$, and $72^{\circ} \mathrm{C}$ for $45 \mathrm{sec}$. The relative number of transcripts for each gene was determined based on linear regression analysis of $100 \mathrm{ng}, 10 \mathrm{ng}$, and $1 \mathrm{ng}$ of M. tuberculosis genomic DNA. The total number of targets $(n)$ were calculated by the equation $n=a$ $+\mathrm{b} \log (\mathrm{x})$ where "a" is the intercept and " $\mathrm{b}$ " is the slope of the standard curve, and " $\mathrm{x}$ " is the threshold cycle obtained by amplifying $\mathrm{n}$ targets. All reactions were performed in triplicate on at least three independent biological replicates.

sigA and $16 \mathrm{~S}$ was monitored to provide additional internal controls.

\section{Acknowledgements}

We gratefully acknowledge Dr. Melissa Ramirez, Dr. Dennis L. Knudson, and Ms. Kerry Brookman for technical and editorial assistance, and Mr. Michael Sherman for assistance with electron microscopy. This work was support by R01 Al055298 (RAS). The funders had no role in study design, data collection and analysis, decision to publish, or preparation of the manuscript.

\section{Author details}

${ }^{1}$ Mycobacteria Research Laboratories, Department of Microbiology, Immunology, and Pathology. Colorado State University, Fort Collins, CO 80523, USA. ${ }^{2}$ Tuberculosis Research Section, NIH/NIAID, 9000 Rockville Pike, Bldg 33, Room 2W20D, Bethesda, Maryland, 20892-3206, USA

\section{Authors' contributions}

KE carried out the experimental studies and RC performed the bioinformatics. RAS designed the studies, and coordination of the manuscript. All authors participated in drafting, and editing the final manuscript. All authors have read and approved the manuscript.

\section{Received: 17 November 2010 Accepted: 19 April 2011}

Published: 19 April 2011

\section{References}

Connolly LE, Edelstein PH, Ramakrishnan L (2007) Why is long-term therapy required to cure tuberculosis? PLoS Med 4(3):e120. doi:10.1371/journal. pmed.0040120

Barry CE, Boshoff HI, Dartois V, Dick T, Ehrt S, Flynn J, Schnappinger D, Wilkinson RJ, Young D (2009) The spectrum of latent tuberculosis: rethinking the biology and intervention strategies. Nat Rev Microbiol 7(12):845-855

Wayne LG (1994) Dormancy of Mycobacterium tuberculosis and latency of disease. Eur J Clin Microbiol Infect Dis 13(11):908-914. doi:10.1007/ BF02111491.

Wayne LG, Hayes LG (1996) An in vitro model for sequential study of shiftdown of Mycobacterium tuberculosis through two stages of nonreplicating persistence. Infect Immun 64(6):2062-2069

Wayne LG (1977) Synchronized replication of Mycobacterium tuberculosis. Infect Immun 17(3):528-530

Slayden RA, Knudson DL, Belisle JT (2006) Identification of cell cycle regulators in Mycobacterium tuberculosis by inhibition of septum formation and global transcriptional analysis. Microbiology 152(Pt 6):1789-1797

Slayden RA, Belisle JT (2009) Morphological features and signature gene response elicited by inactivation of Ftsl in Mycobacterium tuberculosis. J Antimicrob Chemother 63(3):451-457. doi:10.1093/jac/dkn507.
Adams DW, Errington J (2009) Bacterial cell division: assembly, maintenance and disassembly of the Z ring. Nat Rev Microbiol 7(9):642-653. doi:10.1038/ nrmicro2198.

Patru MM, Pavelka MS Jr (2010) A role for the class A penicillin-binding protein PonA2 in the survival of Mycobacterium smegmatis under conditions of nonreplication. J Bacteriol 192(12):3043-3054. doi:10.1128/JB.00025-10.

Hett EC, Rubin EJ (2008) Bacterial growth and cell division: a mycobacterial perspective. Microbiol Mol Biol Rev 72(1):126-156. table of contents. doi:10.1128/MMBR.00028-07.

Trusca D, Scott S, Thompson C, Bramhill D (1998) Bacterial SOS checkpoint protein SulA inhibits polymerization of purified FtsZ cell division protein. $J$ Bacteriol 180(15):3946-3953

Mukherjee A, Cao C, Lutkenhaus J (1998) Inhibition of FtsZ polymerization by SulA, an inhibitor of septation in Escherichia coli. Proc Natl Acad Sci USA 95(6):2885-2890. doi:10.1073/pnas.95.6.2885.

Lutkenhaus J (2007) Assembly dynamics of the bacterial MinCDE system and spatial regulation of the Z ring. Annu Rev Biochem 76:539-562. doi:10.1146/ annurev.biochem.75.103004.142652

Honaker RW, Leistikow RL, Bartek IL, Voskuil MI (2009) Unique roles of DosT and DosS in DosR regulon induction and Mycobacterium tuberculosis dormancy. Infect Immun 77(8):3258-3263. doi:10.1128/IAI.01449-08.

Domenech P, Kolly GS, Leon-Solis L, Fallow A, Reed MB (2010) Massive gene duplication event among clinical isolates of the Mycobacterium tuberculosis W/Beijing family. J Bacteriol 192(18):4562-4570. doi:10.1128/JB.00536-10.

Reed MB, Gagneux S, Deriemer K, Small PM, Barry CE (2007) The W-Beijing lineage of Mycobacterium tuberculosis overproduces triglycerides and has the DosR dormancy regulon constitutively upregulated. J Bacteriol 189(7):2583-2589. doi:10.1128/JB.01670-06.

Respicio L, Nair PA, Huang Q, Anil B, Tracz S, Truglio JJ, Kisker C, Raleigh DP, Ojima I, Knudson DL., et al (2008) Characterizing septum inhibition in Mycobacterium tuberculosis for novel drug discovery. Tuberculosis (Edinb) 88(5):420-429. doi:10.1016/j.tube.2008.03.001.

Huang Q, Kirikae F, Kirikae T, Pepe A, Amin A, Respicio L, Slayden RA, Tonge PJ, Ojima I (2006) Targeting FtsZ for antituberculosis drug discovery: noncytotoxic taxanes as novel antituberculosis agents. J Med Chem 49(2):463-466. doi:10.1021/jm050920y.

Cole ST, Brosch R, Parkhill J, Garnier T, Churcher C, Harris D, Gordon SV, Eiglmeier K, Gas S, Barry CE., et al (1998) Deciphering the biology of Mycobacterium tuberculosis from the complete genome sequence. Nature 393(6685):537-544. doi:10.1038/31159.

Rezwan M, Grau T, Tschumi A, Sander P (2007) Lipoprotein synthesis in mycobacteria. Microbiology 153(Pt 3):652-658

Chauhan A, Lofton H, Maloney E, Moore J, Fol M, Madiraju MV, Rajagopalan M (2006) Interference of Mycobacterium tuberculosis cell division by Rv2719c, a cell wall hydrolase. Mol Microbiol 62(1):132-147. doi:10.1111/j.13652958.2006.05333.x

Chauhan A, Madiraju MV, Fol M, Lofton H, Maloney E, Reynolds R, Rajagopalan M (2006) Mycobacterium tuberculosis cells growing in macrophages are filamentous and deficient in FtsZ rings. J Bacteriol 188(5):1856-1865. doi:10.1128/JB.188.5.1856-1865.2006.

Rustad TR, Sherrid AM, Minch KJ, Sherman DR (2009) Hypoxia: a window into Mycobacterium tuberculosis latency. Cell Microbiol 11(8):1151-1159. doi:10.1111/j.1462-5822.2009.01325.x.

Zhang Y, Hatch KA, Wernisch L, Bacon J (2008) A Bayesian Change point model for differential gene expression patterns of the DosR regulon of Mycobacterium tuberculosis. BMC Genomics 9:87. doi:10.1186/1471-2164-987

Park HD, Guinn KM, Harrell MI, Liao R, Voskuil MI, Tompa M, Schoolnik GK, Sherman DR (2003) Rv3133c/dosR is a transcription factor that mediates the hypoxic response of Mycobacterium tuberculosis. Mol Microbiol 48(3):833-843. doi:10.1046/j.1365-2958.2003.03474.x.

Bartek IL, Rutherford R, Gruppo V, Morton RA, Morris RP, Klein MR, Visconti KC, Ryan GJ, Schoolnik GK, Lenaerts A, Voskuil MI (2009) The DosR regulon of M. tuberculosis and antibacterial tolerance. Tuberculosis (Edinb) 89(4):310-316. doi:10.1016/j.tube.2009.06.001.

Converse PJ, Karakousis PC, Klinkenberg LG, Kesavan AK, Ly LH, Allen SS, Grosset JH, Jain SK, Lamichhane G, Manabe YC., et al (2009) Role of the dosR-dosS two-component regulatory system in Mycobacterium tuberculosis virulence in three animal models. Infect Immun 77(3):1230-1237. doi:10.1128/ |Al.01117-08. 
Murphy DJ, Brown JR (2007) Identification of gene targets against dormant phase Mycobacterium tuberculosis infections. BMC Infect Dis 7:84. doi:10.1186/1471-2334-7-84

Kazmierczak MJ, Wiedmann M, Boor KJ (2005) Alternative sigma factors and their roles in bacterial virulence. Microbiol Mol Biol Rev 69(4):527-543. doi:10.1128/ MMBR.69.4.527-543.2005

Manganelli R, Provvedi R, Rodrigue S, Beaucher J, Gaudreau L, Smith I (2004) Sigma factors and global gene regulation in Mycobacterium tuberculosis. J Bacteriol 186(4):895-902. doi:10.1128/JB.186.4.895-902.2004.

Williams EP, Lee JH, Bishai WR, Colantuoni C, Karakousis PC (2007) Mycobacterium tuberculosis SigF regulates genes encoding cell wallassociated proteins and directly regulates the transcriptional regulatory gene phoY1. J Bacteriol 189(11):4234-4242. doi:10.1128/JB.00201-07.

Michele TM, Ko C, Bishai WR (1999) Exposure to antibiotics induces expression of the Mycobacterium tuberculosis sigF gene: implications for chemotherapy against mycobacterial persistors. Antimicrob Agents Chemother 43(2):218-225

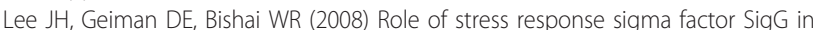
Mycobacterium tuberculosis. J Bacteriol 190(3):1128-1133. doi:10.1128/ JB.00511-07.

Raman S, Song T, Puyang X, Bardarov S, Jacobs WR Jr, Husson RN (2001) The alternative sigma factor $\mathrm{SigH}$ regulates major components of oxidative and heat stress responses in Mycobacterium tuberculosis. J Bacteriol 183(20):6119-6125. doi:10.1128/JB.183.20.6119-6125.2001.

Hu Y, Kendall S, Stoker NG, Coates AR (2004) The Mycobacterium tuberculosis sigJ gene controls sensitivity of the bacterium to hydrogen peroxide. FEMS Microbiol Lett 237(2):415-423

Hahn MY, Raman S, Anaya M, Husson RN (2005) The Mycobacterium tuberculosis extracytoplasmic-function sigma factor SigL regulates polyketide synthases and secreted or membrane proteins and is required for virulence. J Bacteriol 187(20):7062-7071. doi:10.1128/JB.187.20.7062-7071.2005.

Agarwal N, Woolwine SC, Tyagi S, Bishai WR (2007) Characterization of the Mycobacterium tuberculosis sigma factor SigM by assessment of virulence and identification of SigM-dependent genes. Infect Immun 75(1):452-461. doi:10.1128/IAI.01395-06.

doi:10.1186/1471-2180-11-79

Cite this article as: England et al:: Mycobacterium tuberculosis septum site determining protein, Ssd encoded by $r v 3660 c$, promotes filamentation and elicits an alternative metabolic and dormancy stress response. BMC Microbiology 2011 11:79.

\section{Submit your next manuscript to BioMed Central and take full advantage of:}

- Convenient online submission

- Thorough peer review

- No space constraints or color figure charges

- Immediate publication on acceptance

- Inclusion in PubMed, CAS, Scopus and Google Scholar

- Research which is freely available for redistribution

Submit your manuscript at www.biomedcentral.com/submit 